\title{
TRANSISTORS IN PULSE CIRCUITS
}


This book is the fourth in a series by the same author published or to be published in the Philips Technical Library

DIODES AND TRANSISTORS

General theory

AUDIOFREQUENCY TRANSISTORS

RADIOFREQUENCY TRANSISTORS

Amplifiers and oscillators

TRANSISTORS IN PULSE CIRCUITS 


\section{TRANSISTORS IN PULSE CIRCUITS}

G. FONTAINE 
CN. V. Philips' Gloeilampenfabrieken, Eindhoven, 1970

\section{English edition}

(C). V. Philips' Gloeilampenfabrieken, Eindhoven, 1971

Softcover reprint of the hardcover 1st edition 1971 978-0-333-02274-0

All rights reserved. No part of this publication may be reproduced or transmitted, in any form or by any means, without permission

SBN: 333022742

ISBN 978-1-349-00168-2

ISBN 978-1-349-00166-8 (eBook)

DOI 10.1007/978-1-349-00166-8

First published in English by

MACMILLAN PRESS LTD

London and Basingstoke

Associated companies in New York, Toronto, Melbourne,

Dublin, Johannesburg and Madras

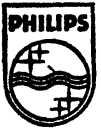

\section{PHILIPS}

Trade marks of N. V. Philips' Gloeilampenfabrieken 


\section{FOREWORD}

With the wider application of electronic systems in industry, and the ever-increasing use of computers, all types of engineers and technicians must be well-informed on the operating principles and the nature of circuits and components.

Information to be produced, transmitted or transformed is now nearer to a square or triangular signal, rather than the sinusoidal oscillations already met with in audio- and radiofrequency. These new signals have their own characteristics, parameters and behaviour patterns, and it is the purpose of this book to study and explain these. Moreover, semiconductors (diodes, transistors, integrated circuits) can distort the functioning of equipment, and a thorough understanding of the basic physical principles involved is necessary to explain these effects and to find ways of reducing or eliminating them.

This book is divided into three main parts: the first deals with factors relating to the purely electrical behaviour of diodes and transistors, and defines the principle 'switching' parameters, i.e. forward and reverse recovery time of the diodes, rise and fall time of the current, delay and desaturation time, etc. The second part outlines the limiting operating conditions of semiconductors by linking the considerations of maximal power and critical temperature. The third and final part of the book is a thorough study of the principal components of switching circuits: astable, monostable and bistable multivibrators, astable and triggered blocking oscillators.

This knowledge is essential for the design, construction and maintenance of components or equipment for numerical and logical systems. For the sake of simplicity, however, we have omitted complex mathematical treatments, as the design of a multivibrator and the definition of its limiting operating conditions can be worked out by very simple equations.

Furthermore, the systematic examination of density graphs enables useful and practical conclusions to be drawn from an apparently qualitative study; a thorough and detailed explanation of all the terms likely to be met in the literature will be found in this book.

August 1969

G. FONTAINE 


\section{CONTENTS}

\section{Chapter 1 - DIODES FOR SWITCHING}

1.1 PN Junction . . . . . . . . . . . . . . . . . . . 2

Difference of impurity concentration . . . . . . . 2

Narrow $\mathrm{N}$ regions . . . . . . . . . . . . 5

1.2 Unbiased Junctions . . . . . . . . . . . . . . . . . . 5

Density graphs on a linear scale . . . . . . . . . . 9

1.3 Biased junctions . . . . . . . . . . . . . . . . 10

Forward biasing . . . . . . . . . . . . . . 10

Reverse bias . . . . . . . . . . . . . . . . 14

1.4 Diodes in switching . . . . . . . . . . . . . . . 18

Switching in the direction of increasing current . . . . 18

Influence of the total resistance of the circuits . . . . . 22

Influence of a sudden variation of voltage . . . . . . . . 25

Note on the use of the networks with static characteristics 29

Definition of the forward recovery time of the diode . . . 30

Influence of a resistance in the circuits . . . . . . . . . 33

Influence of the amplitude of the signal . . . . . . . . . 41

Resistance of a diode . . . . . . . . . . . . . . . 45

Influence of forward bias on the neutrality of the N region 46

Variations of the resistance of a diode as a function of the applied signal . . . . . . . . . . . . . 49

Rise time of the current in the circuit . . . . . . . 50

Influence of the amplitude of the applied signal. . . . . . 54

Switching from forward bias to zero bias . . . . . . . . . 57

Study of static behaviour . . . . . . . . . . . 57

Transient effects . . . . . . . . . . . . . 58

Absence of resistance in the circuit . . . . . . . . . . 58

Definition of the reverse recovery time of the diode . . . . 65

Influence of the resistance of the circuit on the reverse recovery time of the diode . . . . . . . . . 66

Transient behaviour of the diode (with a resistance) . . . 69

Influence of the resistance of the reverse recovery time 73

Change from forward to zero bias . . . . . . . . . . . . 74

Measurement of the reverse recovery time of the diode . . 77

Overall forms of the signals . . . . . . . . . . . . 77 
2.1 Basic physical study . . . . . . . . . . . . . 80

Absence of bias . . . . . . . . . . . . . . . 80

2.2 Operating conditions of a transistor . . . . . . . 83

2.3 Cut-off state . . . . . . . . . . . . . . . . . . . 83

Common-emitter configuration . . . . . . . . . 84

2.4 Normal State . . . . . . . . . . . . . . . . . 87

2.5 Reverse operating state . . . . . . . . . . . . . 91

2.6 Saturation state . . . . . . . . . . . . . . . . 95

2.7 Operating principles of transistors with pulse drive . . . . . 99

2.8 Pulse amplification . . . . . . . . . . . . . . . . . . . 100

Study of an amplifier stage with common-emitter configuration 103

Use of density graphs . . . . . . . . . . . . 103

Influence of the drive on the rise time of the current in the circuit. . . . . . . . . . . . . . . . 104

Influence of the drive on the fall time of the current in the circuit. . . . . . . . . . . . . . . . 107

Quantitative expression of the triggering times . . . . 108

2.9 Thermal instability of transistors . . . . . . . . . . . 111

2.10 Improvement in the shape of the collector current . . . . . 112

Improvement in the rise time of the collector current . . . 112

Improvement in the fall time of the collector current . . . 119

2.11 Definition of the different switching times of a transistor. . . 124

Physical explanation of the switching time . . . . . . . 127

Causes of the delay time of the current . . . . . . . . 127

Significance of the rise time of the current . . . . . . . . 128

Explanation in the delay in the fall time of the current . . . 131

Explanation of the fall time of the current . . . . . . . 135

Quantitative expression for the triggering time . . . . . 136

Advantages and disadvantages of a strong saturation . . . . 139

Advantages and disadvantages of a strong cut-off . . . . . 139

Influence of the fall time . . . . . . . . . . . . . . . 140

2.12 Influence of the type of drive in the large-signal state on the 143

shape of the collector current . . . . . . . . . . . . 143

Effect of saturation on the input resistance of a transistor . . 143

2.13 Speed-up circuit . . . . . . . . . . . . . . . 147

Composition of speed-up circuit . . . . . . . . . . . . 147

Influence of the speed-up circuit on the transient period . . . 147

Use of networks of characteristics and density graphs to in-

dicate the advantages of the speed-up circuit . . . . . . 148

Establishment of the collector current . . . . . . . . . . 151

Desaturation and fall time of the current . . . . . . 151

Expression for the triggering time. . . . . . . . 152 
Explanation of the capacitive effects peculiar to transistors . 156

Diffusion capacitance . . . . . . . . . . . . . 156

Barrier capacitance . . . . . . . . . . . . . 156

Transient capacitance . . . . . . . . . . . . 156

Static equivalent circuit . . . . . . . . . . . 159

Dynamic equivalent circuit . . . . . . . . . . 159

Equivalent circuit in the cut-off state . . . . . . . . . 160

Equivalent circuit in the normal state . . . . . . . 160

Change from cut-off to the normal state . . . . . . . 163

Equivalent circuit in the saturation state . . . . . . 163

Equivalent circuit for the inverse state . . . . . . . . 163

Equivalent switching circuit of a transistor in common emitter 164

2.15 Switching parameters of the transistor . . . . . . . . . 167

Static parameters . . . . . . . . . . . . . 167

Time parameters . . . . . . . . . . . . . . . 168

2.16 Symmetrical transistors . . . . . . . . . . . . 171

Principle of the symmetrical transistor. . . . . . . . . . . 171

Advantages of the symmetrical transistor . . . . . . . . . 172

2.17 NPN transistors . . . . . . . . . . . . . . . 175

Equivalent dynamic 'switching' circuits . . . . . . . . 176

Chapter 3 - INFLUENCE OF THE LOAD

Types of load . . . . . . . . . . . . . . . 180

3.1 Resistive load . . . . . . . . . . . . . . . 183

Permanent normal state . . . . . . . . . . . . 183

Change from the cut-off to the saturation state . . . . . . . 184

Change from the non-conducting to the normal state . . . 187

3.2 Capacitive and resistance load . . . . . . . . . . . . 188

Normal state. . . . . . . . . . . . . . . . 191

Transition from the cut-off to the normal state . . . . . 192

Change from the cut-off to the saturation state . . . . . . 196

3.3 Inductive and resistive load . . . . . . . . . . 200

Normal study . . . . . . . . . . . . . . 203

3.4 Change from the cut-off to the normal state . . . . . . . . 207

3.5 Change from the cut-off state to saturation . . . . . . . 211

Rise of the current . . . . . . . . . . . . . . 211

Disappearance of the current. . . . . . . . . . . 211

Under-damping . . . . . . . . . . . . . 212

Over-damping . . . . . . . . . . . . . 214 
4.1 Power considerations . . . . . . . . . . . . . 216

4.2 Reminder of the thermal behaviour of transistors . . . . . 216

4.3 Power dissipated in a transistor . . . . . . . . . . . . . 224

4.4 Mean power dissipated in the transistor . . . . . . . . . 231

4.5 Calculation of the power dissipated in the transistor . . . 232

Mean power dissipated in the transistor . . . . . . . . 235

Calculation of the amounts of power dissipated during the various stages . . . . . . . . . . . . . . 236

Influence of the lengthening of the switching time . . . . 239

4.6 Resistive load . . . . . . . . . . . . . . . . . . . 240

4.7 Inductive load . . . . . . . . . . . . . . . . 240

4.8 Influence of a diode in parallel with the inductance . . . . 243

4.9 Instantaneous values of the power dissipated in the transistor 244

4.10 Controlled power . . . . . . . . . . . . . . . 244

4.11 Equivalent thermal circuits . . . . . . . . . . . . 252

4.12 Dynamic equivalent thermal circuits. Transient thermal impedance .. . . . . . . . . . . . . . 255

4.13 Influence of the transient thermal impedance . . . . . . 256

4.14 Instantaneous junction temperature . . . . . . . . . 260

4.15 Thermal rise time constant . . . . . . . . . . . . 264

Thermal time constant short by comparison with the pulse duration. . . . . . . . . . . . . . . . 264

Shorter pulses and those with a higher recurrence frequency 264

Signals of short duration by comparison with their spacing . 267

Pulses of long duration with respect to their separation . . . 268

Time for the establishment of the steady state . . . . . . . 271

Diagrammatic method for the determination of the maximum temperature of the junction . . . . . . . . 272

Chapter 5 - SWITCHING CIRCUITS AND MULTIVIBRATORS

5.1 Principle of switching . . . . . . . . . . . . . . . . . 278

Production of a rectangular signal . . . . . . . . . 278

Diagrammatic determination of the influence of the resistance

R ............... 281

Resemblance of a diode to a switch . . . . . . . . 281

Resemblance of a transistor to two switches . . . . . . 281

5.1 Influence of a contact resistance . . . . . . . . . . 282

Influence of a leakage resistance . . . . . . . . 285

Effect of inertia . . . . . . . . . . . . 285 
5.2 Transistor in common emitter. Switching function . . . . 286 Representation of the input circuit . . . . . . . . 286

Representation of the output circuit. . . . . . . . . 289

Rapid switching . . . . . . . . . . . . 290

Chapter 6 - PRINCIPAL SWITCHING CIRCUITS

6.1 Chief functions of switching circuits. . . . . . . . . . 294

6.2 Generation of signals . . . . . . . . . . . . . 294

6.3 Pulse shaping . . . . . . . . . . . . . . . . . 294

6.4 Counting . . . . . . . . . . . . . . . . . . . . 297

6.5 Similarity of the three types of multivibrator . . . . . . . 298

6.6 Other switching circuits . . . . . . . . . . 298

\section{Chapter 7 - ASTABLE MULTIVIBRATORS}

7.1 Theory and operating principle of an astable multivibrator . 304

7.2 Diagrammatic representation of the signals . . . . . . 308

First pseudostable state . . . . . . . . . . . . 311

Second pseudostable state . . . . . . . . . . . 312

Influence of the time constants on the shape and duration of the signal ................ 315

RC Circuits with long time constants . . . . . . . . 316

7.3 Calculation of the instantaneous values of current and voltage 319

Static operating conditions. . . . . . . . . . 319

Dynamic operating conditions . . . . . . . . . 320

Respect of the cut-off and saturation states . . . . . . 331

Rapid switching ............... . 331

7.4 Calculation of powers . . . . . . . . . . . . 332

7.5 Limiting values of the voltage and currents . . . . . . . 335

7.6 Signal shaping at the output . . . . . . . . . . . 336

Use of diodes in the collectors of the transistors . . . . . 336

Use of diodes associated with resistors . . . . . . . 339

7.7 Actual circuits . . . . . . . . . . . . . . . . . 340

Astable multivibrator with two capacitive couplings . . . 340

Astable multivibrator with emitter coupling . . . . . . 340

Chapter 8 - THE MONOSTABLE MULTIVIBRATOR

8.1 Concept and principle of operation of a monostable multivibrator . . . . . . . . . . . . . . 344

8.2 Calculation of the values of voltages and currents in the steady state 
8.3 Physical explanation of the operator with diagrammatic presentation of the signals . . . . . . . . . . 355

8.4 Calculation of the voltages and currents during the dynamic period . . . . . . . . . . . . . . . 359

8.5 Correction circuit. . . . . . . . . . . . . . 363

8.6 Problems concerned with the triggering of a Monostable multivibrator .............. 363

8.7 An actual circuit . . . . . . . . . . . . 367

Chapter 9 - THE BISTABLE MULTIVIBRATOR

9.1 Concept and principle of operation of a bistable multivibrator 370

9.2 Calculation of the values of the voltages and currents for the two stable states .............. 373

9.3 Diagrammatic representation. . . . . . . . . . . . 382

9.4 Diagrammatic method for the design of a bistable multivibrator. . . ........... 386

9.5 Triggering of a bistable multivibrator . . . . . . . 402

9.6 Actual circuit . . . . . . . . . . . . . 409

Chapter 10 - SWITCHING CIRCUITS BLOCKING OSCILLATORS

10.1 Astable blocking oscillators . . . . . . . . . . . . 412

10.2 Astable blocking oscillator with an RC circuit in the base . . 423

10.3 Astable blocking oscillator with RC circuit in series . . . . 427

10.4 Astable blocking oscillator . . . . . . . . . . . . . . 428

10.5 Triggered blocking oscillators . . . . . . . . . . . . 431

10.6 Triggered blocking oscillator with feedback between the collector and base . . . . . . . . . . . 4 436

10.7 Triggered blocking oscillator excited by the base . . . . . . 447

10.8 Triggered blocking oscillator with biasing circuit in the emitter 447

10.9 Triggered blocking oscillator with feedback between the collector and emitter . . . . . . . . . . . . 448 